\title{
CURVE-OF-GROWTH ANALYSIS OF A RED GIANT IN M 67
}

\author{
R. GRIFIIN \\ Cambridge University, U.K.
}

\begin{abstract}
A coudé spectrogram of a red giant (IV-202) in M 67 has been analyzed with respect to Arcturus by the differential curve-of-growth method. The overall metal abundance is found to be approximately twice that in Arcturus, i.e. half the solar value. The error limits exclude the interpretation that IV-202 is 'super-metal-rich'. According to other observational evidence, IV-202 is quite typical of the red giants in M 67 .
\end{abstract}

\section{Reference}

Griffin, R.: 1975, Monthly Notices Roy. Astron. Soc. 171, 181.

\section{DISCUSSION}

Bidelman: Could you give us a more conventional or more respectable designation for this object?

Griffin: It is BD $+12^{\circ} 1919$.

Williams: Could you tell us a bit more about your determination of the temperature of this star particularly whether you have any feel for the line temperature (e.g. $\Delta \theta_{\text {ex }}$ ) and how it compares with $\alpha$ Tau.

Griffin: First I derived a rough value of $\Delta \theta$ ex from partial curves of growth. (I had to assume that differential excitation and effective temperatures would be identical). Then, accepting the value $\Delta \theta=+0.11$ for ( $\alpha$ Tau $-\alpha$ Boo) given recently by van Paradijs and Meurs, I turned to Eggen's photometry and interpolated to find the corresponding differential temperature for IV-202.

Osborn: What is the approximate bolometric magnitude of this star?

Griffin: The value given by Eggen is -1.75 .

Spinrad: Indeed, M 1465 = IV-202 in M67, from ST scans still has the visual M 67 anomaly-strong resonance lines of $\mathrm{Ca}, \mathrm{Mg}, \mathrm{NaI}$. These lines are stronger than the normal field giant of a similar red colour.

Griffin: I believe the enhancement of such lines might be expected in a star that is both as cool and as luminous as IV-202; its estimated spectral type is $\mathrm{K} 3$ or $\mathrm{K} 4$, luminosity class II-III. 